\title{
Preliminary Study of Nondestructive Testing of the Polymer Cutoff Wall Based on Vibration Theory
}

\author{
Shu Zhang, ${ }^{1}$ Binghan Xue $\mathbb{D}^{1,2}$ Jing Wang, ${ }^{1}$ Jianglin Gao, ${ }^{3}$ Chaojie Wang, \\ and Wenzhong $\mathrm{Li}^{4}$ \\ ${ }^{1}$ School of Water Conservancy Engineering, Zhengzhou University, Zhengzhou 450001, China \\ ${ }^{2}$ BESTDR Infrastructure Hospital (Pingyu), Zhumadian 463000, China \\ ${ }^{3}$ Jiangxi Academy of Water Science and Engineering, Nanchang 330000, China \\ ${ }^{4}$ Changjiang Institute of Survey, Planning,Design and Research, Wuhan 430010, China
}

Correspondence should be addressed to Binghan Xue; xuebinghan@zzu.edu.cn

Received 9 June 2021; Accepted 2 August 2021; Published 11 August 2021

Academic Editor: Zhengyang Song

Copyright (C) 2021 Shu Zhang et al. This is an open access article distributed under the Creative Commons Attribution License, which permits unrestricted use, distribution, and reproduction in any medium, provided the original work is properly cited.

The polymer cutoff wall offers the benefits of causing little disturbance to the dam body, being convenient and economical to construct and practical to use, and possessing good resistance to seismic shocks and cracks. It has been widely used to prevent seepage and to reinforce dikes and dams. However, the polymer cutoff wall is a concealed underground structure, and nondestructive testing methods to ensure its integrity are not yet mature. This paper describes a modal analysis of polymer cutoff wall models with different damage scenarios to investigate the feasibility of nondestructive testing of the polymer cutoff wall based on vibration theory. The dynamic characteristics of the first three natural frequencies of the wall and their mode shapes show that horizontal direction damage and centrally located partial damage have a noticeable impact on the dynamic characteristics of the wall, indicating that nondestructive testing based on vibration theory is useful to test for horizontal damage and moderate damage located centrally in the wall.

\section{Introduction}

China has built about 98,000 reservoirs: about 4700 large and medium-sized reservoirs and more than 94,000 small reservoirs. The total length of the embankments is over $410,000 \mathrm{~km}$. Most of this water conservancy infrastructure was built between 1950 and 1970. Some embankments had inherent defects due to the low level of available technology and economic austerity in that period. Long-term disrepair, flooding, earthquakes, and other natural disasters have made these reservoirs sources of disease. Any reservoir accident is likely to severely affect human security, leading to immeasurable loss of life and property damage $[1,2]$.

Nonaqueous reaction polymer grouting is a new antiseepage technology that has been developed to reinforce soil dikes and dams to prevent seepage [3]. It is a product of the National Water Conservancy Advanced Practical Technology Key Promotion project. This technology adopts the construction technique of static pressure groove formation, lifting grouting, and sleeve hole connections to realize the simultaneous construction of pressure groove grouting. Polymer cutoff walls provide many significant advantages over cement or concrete cutoff walls [4]: they can be rapidly and accurately constructed, there is little disturbance to the dam when they are installed, they are economical and practical in use, and they have good resistance to seismic activity, cracking, and corrosion [5].

The integrity of a polymer cutoff wall is important for the safe operation of the dam. The polymer cutoff wall is underground and therefore concealed. Thus, nondestructive testing of the polymer cutoff wall is a necessity. However, current research on polymer cutoff walls is mainly concerned with their static and dynamic properties [6-9], and there are few studies of nondestructive testing methods for polymer cutoff walls [10]. One nondestructive testing method, which is based on vibration theory, assesses the 
integrity of a cutoff wall by determining changes in modal or structural parameters [11]. This method provides the benefits of maintaining structural integrity, rapid damage detection, and low cost and can be used for large-scale inspections. However, the applicability of nondestructive testing methods based on vibration theory to thin and lightweight polymer cutoff walls has not been conclusively demonstrated [12].

Nondestructive testing based on vibration theory is a global method that requires the measurement of modal parameters of the system under investigation, such as natural frequency and mode shape. The underlying paradigm is that modal parameters of a system are a function of the physical properties of the system (such as stiffness, mass, and damping), so changes in the physical properties of the structure will lead to corresponding changes in the modal parameters of the structure [13]. Conversely, changes in modal parameters can also indicate changes in physical structural properties [14]. Lifshite and Rotem initially proposed that structural damage could be detected by observing changes in the natural frequency of the structure [15]. The method they introduced has been widely used for large civil engineering structures in fields such as aviation, aerospace, and precision machinery and for oil drilling platforms, large bridges, super-high-rise buildings, and large span grids [16]. Li analyzed the parameters and modeled the dynamics of vibrations in a damaged bridge structure in multiple steps. The author first created a model of the entire bridge and identified the approximate location of the structural damage and then quantified the extent of the damage. The study accurately identified and quantified the damage [17]. Liu and He demonstrated the feasibility and effectiveness of the use of the frequency ratio of a structure as a diagnostic index of structural damage through experimental tests of straight steel pipes, steel shells, and a 5-story steel frame structure [18]. Cheng et al. investigated the sensitivity to structural damage of several mode indicators of typical gravity dams through numerical modeling and physical experiments. They found that, of the parameters they investigated, COMAC and the Lipschitz index were the most sensitive to structural damage [19].

In this paper, we propose a modal analysis of various states of polymer cutoff walls: intact, horizontally damaged, vertically damaged, and partially centrally damaged. We compare the first three natural frequencies and modes of each damaged cutoff wall with those of the intact cutoff wall. Our investigation of the applicability of nondestructive testing methods based on vibration theory for the polymer cutoff wall will provide a theoretical basis for future nondestructive testing of polymer cutoff walls.

\section{Modal Analysis of a Polymer Cutoff Wall}

The principle of nondestructive frequency testing is to apply a certain level of excitation to cause the structure to vibrate and then to instrumentally measure the excitation force and the responses of the structure, such as displacement, velocity, and acceleration. The modal characteristics of the structure are then obtained by digital signal processing techniques, and the damage is then determined according to changes in the modal parameters of the structure. Thus, nondestructive testing enables us to observe changes in the modal parameters of the polymer cutoff wall to determine whether it is damaged. The quality and stiffness of the damaged polymer cutoff wall will change its natural frequency, and this change will be observed in the detection of vibration and the vibration mode. Thus, it is necessary to investigate vibration mode changes between the intact and damaged polymer cutoff walls.

2.1. Calculation Model. Each section of the polymer cutoff wall of the subject dam was $10 \mathrm{~m}$ long, its width was $0.03 \mathrm{~m}$, and its depth was $15 \mathrm{~m}$. The polymer material used in the wall had density $\rho=240 \mathrm{~kg} / \mathrm{m}^{3}$, elastic modulus $E=180 \mathrm{MPa}$, and Poisson ratio $\mu=0.20$. The polymer cutoff wall was modeled by SOLID 185 element. The soil density of the dam was $\rho=1740 \mathrm{~kg} / \mathrm{m}^{3}$, the shear wave (transverse wave) velocity was $V_{s}=241 \mathrm{~m} / \mathrm{s}$, and the compression wave (horizontal wave) velocity was $V_{p}=439 \mathrm{~m} / \mathrm{s}$, determined by onsite wave velocity measurement.

There is interaction between a polymer cutoff wall and the surrounding soil, so the influence of the surrounding soil on the polymer cutoff wall should be considered when modal analysis is undertaken to calculate the dynamic characteristics of the polymer cutoff wall. The effects of the soil on the polymer cutoff wall can be modeled by equivalent stiffness springs. In this paper, COMBIN 14 element is selected for the establishment of spring unit. Equivalent stiffness is determined by extension of the equivalent stiffness of the pile foundation. The equivalent stiffness [11] is

$$
K=\frac{2.75 G_{d} A_{1}}{L}+\frac{8 G_{d} A_{2}}{\left(1-\mu_{d}\right) L},
$$

where $G_{d}$ is the dynamic shear modulus of the surrounding soil, $\mu_{d}$ is the Poisson ratio of the surrounding soil, $A_{1}$ is the side area of the polymer cutoff wall, $A_{2}$ is the bottom area of the polymer cutoff wall, and $L$ is the perimeter of the bottom of the polymer cutoff wall.

The parameters $A_{1}, A_{2}$, and $L$ are determined by the actual size of the polymer cutoff wall. $G_{d}$ and $\mu_{d}$ are determined by the following equations [20]:

$$
\begin{aligned}
G_{d} & =\rho V_{s}^{2}, \\
\mu_{d} & =-\frac{1 / 2\left(V_{p} / V_{s}\right)^{2}-1}{\left(V_{p} / V_{s}\right)^{2}-1},
\end{aligned}
$$

where $\rho$ is the density of the soil medium around the polymer cutoff wall, $V_{s}$ is the shear wave velocity of the soil medium around the polymer cutoff wall, and $V_{p}$ is the compression wave velocity of the soil medium around the polymer cutoff wall.

The polymer cutoff wall sections were connected to each other relatively firmly using sleeve connections. The boundaries between polymer cutoff walls were, therefore, considered to be fixed. The two sides of the polymer cutoff wall were in contact with the soil, were considered to be 
infinite, and were perpendicular to the direction of vibration excitation. It was, therefore, assumed that the cutoff wall was not displaced in this direction. The natural frequency of higher order vibrations is short, the natural vibration period is low, and any impact on the structure was difficult to observe [21]. Thus, in the modal analysis of the polymer cutoff wall, only the first three natural frequencies and natural vibration modes of the polymer cutoff wall were considered.

In modeling damage on the polymer cutoff wall, three different damage scenarios were considered: in the horizontal direction, in the vertical direction, and in the central part of the wall. For each type of damage, we considered both mild and moderate damage. The horizontal length of the intact polymer cutoff wall was $L_{0}$, its vertical height was $H_{0}$, and the length and height of the damage to the wall were, respectively, $L_{n}$ and $H_{n}$.

\subsection{Calculation Results}

2.2.1. Intact Polymer Cutoff Wall. The intact polymer cutoff wall model consisted of 287,200 units and 96,907 nodes. Equation (1) gives the equivalent stiffness of the soil surrounding the intact impermeable wall as $K=4.1732 \mathrm{GN} / \mathrm{m}$. Modal analysis calculated the first 3 natural frequencies of the intact polymer cutoff wall, which are shown in Table 1. The shapes of the first three modes are shown in Figure 1.

\subsubsection{Horizontal Damage to Polymer Cutoff Wall. For slight} damage to the polymer cutoff wall in a horizontal direction, we supposed that the polymer cutoff wall had separated at a distance of $1 \mathrm{~m}$ from the boundary. In this scenario, the horizontal length of the polymer cutoff wall $L_{1}=9 \mathrm{~m}$. There were 254710 units and 85705 nodes in the model. Equation (1) gave an equivalent stiffness of the soil surrounding the intact impermeable polymer wall as $K=4.10303 \mathrm{GN} / \mathrm{m}$. For moderate damage to the wall, we supposed that it had separated at a distance of $L_{0} / 4 \mathrm{~m}$ from the boundary and that the length of the damaged polymer cutoff wall $L_{2}=7.5 \mathrm{~m}$. There were 206381 units and 69549 nodes in the model. Equation (1) gave an equivalent stiffness of the soil surrounding the intact impermeable polymer wall as $K=4.1003 \mathrm{GN} / \mathrm{m}$. Because the entire wall was broken, the fracture surface was no longer constrained by adjacent wall sections. In the two horizontal damage cases, modal analysis gave the first three natural frequencies of the horizontally damaged polymer cutoff wall that are shown in Table 2; the first three natural frequency modes are shown in Figure 2 and Figure 3.

2.2.3. Vertical Damage to Polymer Cutoff Wall. For slight damage to the polymer cutoff wall in the vertical direction, we supposed that the wall had separated vertically for a distance of $1 \mathrm{~m}$ starting at the bottom of the wall and that the height of the wall was $H_{1}=14 \mathrm{~m}$. There were 270404 units and 90874 nodes in the model. Equation (1) gave the equivalent stiffness of the soil surrounding the intact
TABLE 1: First three natural frequencies of the intact polymer cutoff wall.

\begin{tabular}{lccc}
\hline Frequency & 1st & 2nd & 3rd \\
\hline$\omega(\mathrm{Hz})$ & 29.989 & 45.071 & 46.035 \\
\hline
\end{tabular}

impermeable wall as $K=3.8319 \mathrm{GN} / \mathrm{m}$. For moderate damage to the wall, we supposed the wall had separated at the bottom for a height $H_{0} / 4$. The height of the wall was $H_{2}=12.25 \mathrm{~m}$. There were 284847 units and 72542 nodes in the model. Equation (1) calculated the equivalent stiffness of the soil surrounding the wall as $K=3.0825 \mathrm{GN} / \mathrm{m}$. Modal analysis of these two scenarios showed the first three natural frequencies of the polymer cutoff wall with vertical damage to those shown in Table 3. The first three-mode shapes are shown in Figures 4 and 5.

\subsubsection{Partially Centrally Damaged Polymer Cutoff Wall.} For partial damage at the center of the polymer cutoff wall, we supposed that a circular area of soil with a diameter of $1 \mathrm{~m}$ was incorporated in an area at the center of the wall. The polymer material in this area was replaced by the surrounding soil. There were 298242 units and 98418 nodes in this model. Equation (1) calculated the equivalent stiffness of the soil surrounding the wall as $K=4.1044 \mathrm{GN} / \mathrm{m}$. For moderate damage to the wall, we supposed there was a circular area of soil with a diameter of $L_{0} / 4 \mathrm{~m}$ in the center of the wall. There were 215500 units and 94259 nodes in the model. Equation (1) calculated the equivalent stiffness of the soil surrounding the wall as $K=4.1044 \mathrm{GN} / \mathrm{m}$. Modal analysis showed that the first three natural frequencies of the partially damaged wall are those shown in Table 4 . The first three-mode shapes are shown in Figures 6 and 7.

\section{Analysis of Dynamic Characteristics}

Table 2 shows that, for the polymer cutoff wall that was damaged horizontally, the first three natural frequencies differed greatly from those for the intact wall. In the case of mild damage, the first three natural frequencies decreased, respectively, by $38 \%, 44 \%$, and $40 \%$. In the case of moderate damage, the respective decreases were $30 \%, 44 \%$, and $40 \%$. A comparison of Figures 2 and 3 with Figure 1 clearly shows that there was a great change in the horizontally damaged wall. Therefore, any one of the first three modes can be used to determine the integrity of a polymer cutoff wall with damage in the horizontal direction.

Table 3 shows that the first three natural frequencies of the polymer cutoff wall were within a small range. In the case of mild damage, the first three natural frequencies increased, respectively, by $1 \%, 0 \%$, and $0 \%$. In the case of moderate damage, the first three natural frequencies increased, respectively, by $7 \%, 0 \%$, and $3 \%$. A comparison of Figures 4 and 5 with Figure 1 shows that the polymer cutoff wall with damage in the vertical direction was similar in terms of frequency to the three-mode diagram of the intact wall, which makes the damaged wall almost indistinguishable from the intact wall. Thus, this method is not sensitive to 


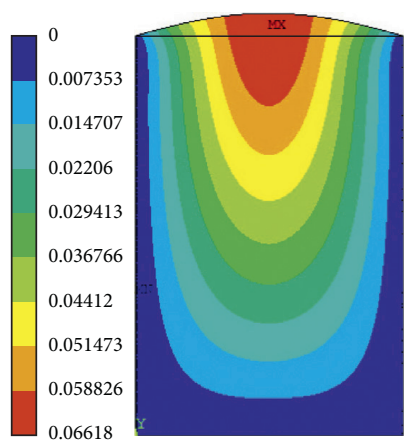

(a)

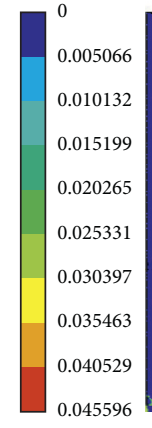

(b)

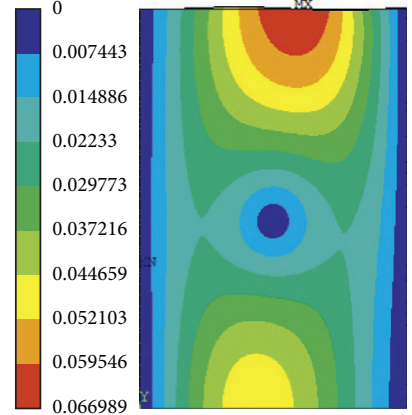

(c)

Figure 1: Shapes of the first three modes of the intact polymer cutoff wall. (a) First vibration mode. (b) Second vibration mode. (c) Third vibration mode.

TABLE 2: First three natural frequencies of the horizontally damaged polymer cutoff wall.

\begin{tabular}{lccccc}
\hline Frequency & Intact $\omega(\mathrm{Hz})$ & Slight damage $\omega(\mathrm{Hz})$ & $\omega_{1}-\omega / \omega(\%)$ & Moderate damage $\omega(\mathrm{Hz})$ & $\omega_{2}-\omega / \omega(\%)$ \\
\hline $1^{\text {st }}$ & 28.89 & 18.681 & -38 & 20.94 & -30 \\
$2^{\text {nd }}$ & 45.071 & 25.211 & -44 & 30.269 & -33 \\
$3^{\text {rd }}$ & 46.035 & 27.431 & -40 & 31.927 & -31 \\
\hline
\end{tabular}

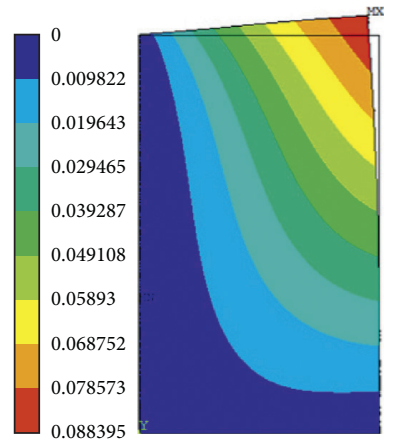

(a)

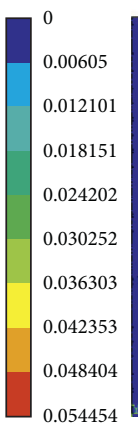

(b)

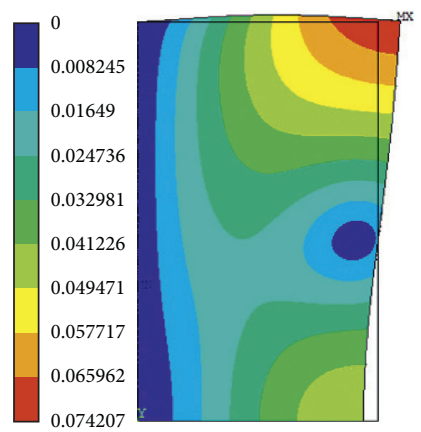

(c)

FIGURE 2: Shapes of the first three modes of the slightly horizontally damaged polymer cutoff wall. (a) First vibration mode. (b) Second vibration mode. (c) Third vibration mode.

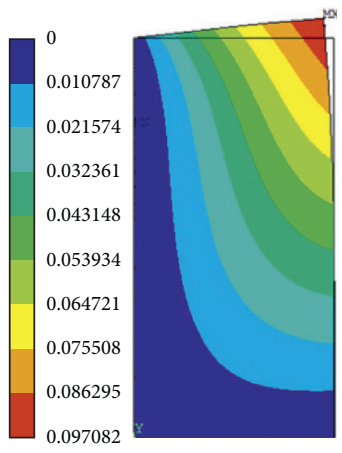

(a)

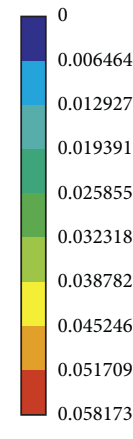

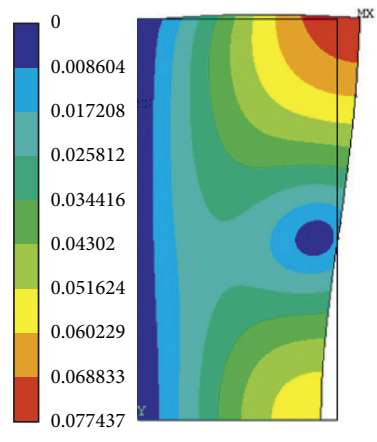

(c)

Figure 3: Shapes of the first three modes of the moderately horizontally damaged polymer cutoff wall. (a) First vibration mode. (b) Second vibration mode. (c) Third vibration mode. 
TABLE 3: First three natural frequencies of the vertically damaged polymer cutoff wall.

\begin{tabular}{lccccc}
\hline Frequency & Intact $\omega(\mathrm{Hz})$ & Slightly damaged $\omega_{3}(\mathrm{~Hz})$ & $\omega_{3}-\omega / \omega(\%)$ & Moderate damaged $\omega_{4}(\mathrm{~Hz})$ & $\omega_{4}-\omega / \omega(\%)$ \\
\hline $1^{\text {st }}$ & 28.989 & 30.41 & 1 & 32.197 \\
$2^{\text {nd }}$ & 45.071 & 45.028 & 0 & 44.874 \\
$3^{\text {rd }}$ & 46.035 & 46.251 & 0 & 47.195 \\
\hline
\end{tabular}

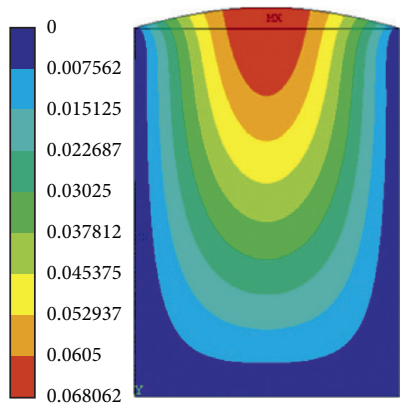

(a)

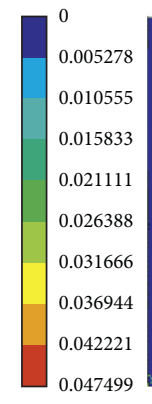

047499

(b)

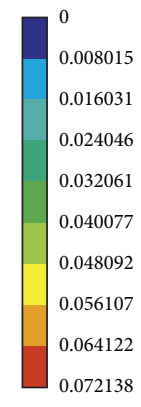

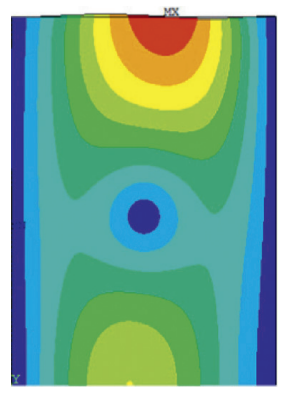

(c)

FIGURE 4: Shapes of the first three modes of the slightly vertically damaged polymer cutoff wall. (a) First vibration mode. (b) Second vibration mode. (c) Third vibration mode.

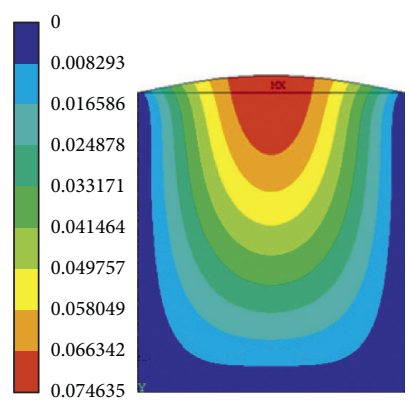

(a)

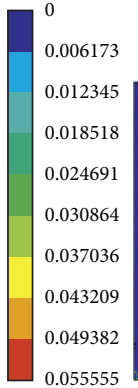

0.055555

(b)

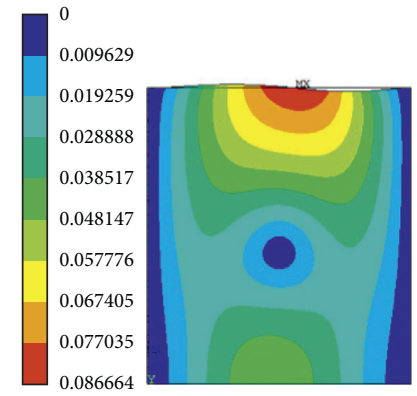

(c)

FIGURE 5: Shapes of the first three modes of the moderately vertically damaged polymer cutoff wall. (a) First vibration mode. (b) Second vibration mode. (c) Third vibration mode.

TABLE 4: First 3-order natural frequencies of polymer cutoff wall with damage in the middle part.

\begin{tabular}{|c|c|c|c|c|c|}
\hline Frequency & Intact $\omega(\mathrm{Hz})$ & Slightly damaged $\omega_{5}(\mathrm{~Hz})$ & $\omega_{5}-\omega / \omega(\%)$ & Moderate damaged $\omega_{6}(\mathrm{~Hz})$ & $\omega_{6}-\omega / \omega(\%)$ \\
\hline $1^{\text {st }}$ & 28.989 & 29.154 & -3 & 24.838 & -17 \\
\hline $2^{\text {nd }}$ & 45.071 & 42.688 & -5 & 31.862 & -29 \\
\hline $3^{\text {rd }}$ & 46.035 & 46.019 & -0 & 41.642 & -10 \\
\hline
\end{tabular}

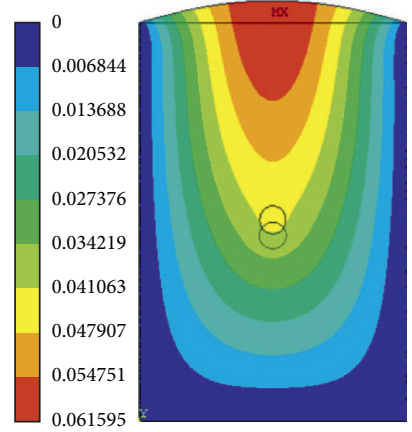

(a)

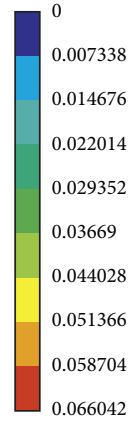

66042

(b)

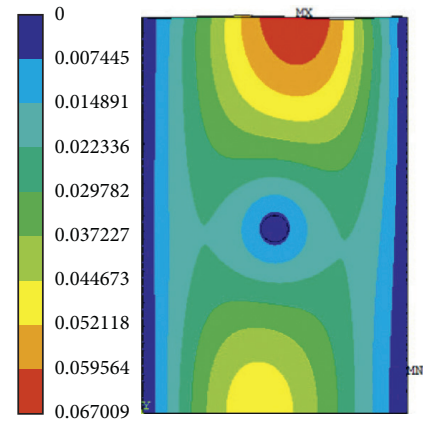

(c)

FIgURE 6: First 3-order mode shapes of polymer cutoff wall with slight damage in the middle part. (a) First vibration mode. (b) Second vibration mode. (c) Third vibration mode. 


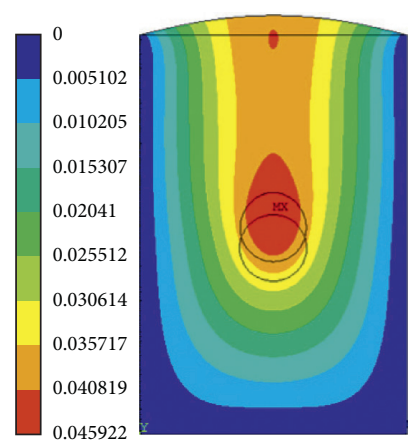

(a)

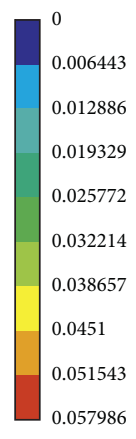

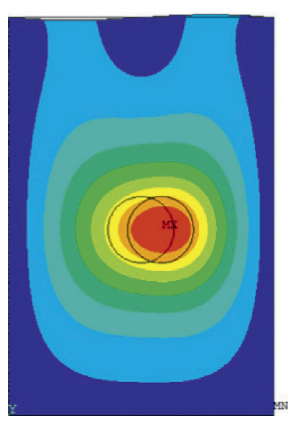

(b)

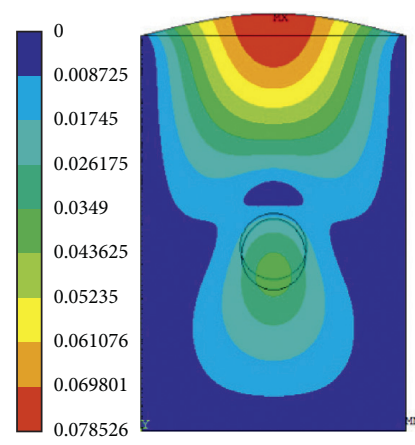

(c)

FIgURe 7: First 3-order mode shapes of polymer cutoff wall with moderate damage in the middle part. (a) First vibration mode. (b) Second vibration mode. (c) Third vibration mode.

vertical damage to the polymer cutoff wall and is therefore not suitable for indicating the integrity of the wall.

Table 4 shows that, in the case of minor local damage to the center of the wall, the first three natural frequencies of the polymer cutoff wall decreased, respectively, by $3 \%, 5 \%$, and $0 \%$. In the case of moderate local damage, the first three natural frequencies decreased, respectively, by $17 \%, 29 \%$, and $10 \%$. Thus, any one of the first three modes can be used to indicate the integrity of the partially damaged polymer cutoff wall when the damage is large.

\section{Conclusion}

In this paper, through modal analysis of the polymer cutoff wall, using the intact, horizontally damaged, vertically damaged, and partly centrally damaged polymer cutoff wall as the basic models, the first three natural frequencies and natural modes of the three models were analyzed and compared with the model of the intact wall. The vibration shape shows that nondestructive testing methods based on vibration theory can be used to indicate the integrity of polymer cutoff walls, but they are individually sensitive to different types of damage.

(1) When the polymer cutoff wall was horizontally damaged, the images of the first three natural frequencies and modes of the damaged polymer cutoff wall were quite different from those of the intact polymer cutoff wall. Any of the first three modes will indicate the integrity of the wall.

(2) When the polymer cutoff wall was vertically damaged, the first three natural frequencies and the three-mode diagrams of the damaged polymer cutoff wall do not change significantly from those of the intact wall, and the sensitivity to this condition is not high.

(3) When the polymer cutoff wall is damaged in the center, the first three natural frequencies of the damaged polymer cutoff wall and the range of the three modes will increase as the damage increases. The sensitivity of this method also increases as the degree of damage increases.

\section{Data Availability}

The data used to support the findings of the study are available from the corresponding author upon request.

\section{Conflicts of Interest}

The authors declare that they have no conflicts of interest.

\section{Acknowledgments}

This work was supported by the National Key Research and Development Program of China (Grant no. 2017YFC1501204), the Natural Science Foundation of Henan Province (Grant no. 212300410279), and the Open Research Fund of Water Engineering Materials and Structures, Guangxi Institute of Water Resources Research (Grant no. GXHRI-WEMS-2020-04). The authors would like to thank for these financial supports.

\section{References}

[1] N. H. Ru and Y. G. Niu, Embankment Dam Incidents and Safety of Large Dams, China Water \& Power Press, Beijing, China, 2001.

[2] Y. N. Bai, X. F. Sun, and J. Bai, "Causes of seepage failure of earth dams and treatment techniques," Water Resources and Hydropower Engineering, vol. 10, pp. 15-16, 2002.

[3] F. M. Wang, J. Li, M. S. Shi, and C. Guo, "New seepage-proof and reinforcing technologies for dikes and dams and their applications," Journal of Hydroelectric Engineering, vol. 12, pp. 1-11, 2016.

[4] X. Cai, Z. Zhou, H. Zang, and Z. Song, "Water saturation effects on dynamic behavior and microstructure damage of sandstone: p," Engineering Geology, vol. 276, Article ID 105760, 2020.

[5] X. Du, H. Fang, S. Wang, B. Xue, and F. Wang, "Experimental and practical investigation of the sealing efficiency of cement grouting in tortuous fractures with flowing water," Tunnelling and Underground Space Technology, vol. 108, Article ID 103693, 2020.

[6] M. S. Shi, F. M. Wang, H. Liu, and C. Guo, "Finite-element simulation and experiment on polymer directional fracturing and grouting for dykes and dams," Journal of Hydraulic Engineering, vol. 8, pp. 1087-1092, 2016. 
[7] J. G. Xu, Z. Fang, B. Wang et al., "Seepage field and stress field coupling analysis of dam with polymer anti-seepage wall," Journal of Water Resources and Architectural Engineering, vol. 4, pp. 1-5, 2017.

[8] J. Li, J. Zhang, J. Xu, and F. Wang, "Dynamic behavior of polymer antiseepage wall for earth dam by centrifuge test," International Journal of Geomechanics, vol. 18, no. 12, pp. 4018171-4018179, 2018.

[9] F. M. Wang, M. J. Li, H. Y. Fang, and B. H. Xue, "Analysis of polymer cut-off wall of yellow river dyke," Yellow River, vol. 41 , no. 10 , pp. 48-52, 2019.

[10] J. G. Xu, C. Y. Zhang, B. Wang, and L. Yan, "Study on application of high-density electrical method to detection of high polymer cut-off wall inearthfill embankment," Water Resources and Hydropower Engineering, vol. 49, no. 12, pp. 72-79, 2018.

[11] S. S. Wang, Research on Nondestructive Testing Technology of Hydraulic Structure Based on Vibration Theory, Hohai University, Nanjing, China, 2005.

[12] W.-X. Ren and G. De Roeck, "Structural damage identification using modal data. I: simulation verification," Journal of Structural Engineering, vol. 128, no. 1, pp. 87-95, 2002.

[13] G. R. Yan, Z. D. Duan, and J. P. Ou, "Review on structural damage detect ion based on vibration data," Earthquake Engineering and Engineering Dynamics, vol. 3, pp. 95-103, 2007.

[14] J. K. Liu and K. Tang, "Advances of vibration based damage identification methods," Acta Scientiarum Naturalium Universitatis Sunyatseni, vol. 43, no. 6, pp. 57-61, 2004.

[15] J. M. Lifshitz and A. Rotem, "Determination of reinforcement unbonding of composites by a vibration technique," Journal of Composite Materials, vol. 3, no. 3, pp. 412-423, 1969.

[16] Z. Zhu, Study on the Theory and Method of Vibration Based Structure Damage Identification, Tsinghua University, Beijing, China, 2006.

[17] R. Li, Based on the Dynamic Characteristics of the Bridge Damage Identification Research, Chang' an University, Shaanxi, China, 2009.

[18] H. B. Liu and F. He, "Study on small damage detection method based on structural dynamic features," World Earthquake Engineering, vol. 30, no. 1, pp. 69-76, 2014.

[19] L. Cheng, Q. L. Qin, and F. Tong, "Sensitivity study on damage diagnosis modal index of hydraulic concrete structure," Earthquake Engineering and Engineering Dynamics, vol. 38, no. 6, pp. 126-136, 2018.

[20] G. T. Meng, The Mechanism, Method and Engineering Application of Soil In-Situ Testing, Geological Publishing House, Beijing, China, 1997.

[21] X. Cai, Z. Zhou, and X. Du, "Water-induced variations in dynamic behavior and failure characteristics of sandstone subjected to simulated geo-stress," International Journal of Rock Mechanics and Mining Sciences, vol. 130, Article ID 104339, 2020. 